\title{
HST Imaging of New Edge-on Circumstellar Disks in Nearby Star-forming Regions
}

\author{
K.R. Stapelfeldt ${ }^{1}$, G. Duchêne ${ }^{2,3}$, M. Perrin ${ }^{4}$, S. Wolff ${ }^{5}$, \\ J.E. Krist ${ }^{6}$, D.L. Padgett ${ }^{1}$, F. Ménard ${ }^{7,3}$, and C. Pinte ${ }^{7,3}$ \\ ${ }^{1}$ NASA GSFC, ${ }^{2}$ UC Berkeley, ${ }^{3}$ IPAG/Grenoble, ${ }^{4}$ STScI, ${ }^{5}$ JHU, \\ ${ }^{6} \mathrm{JPL} /$ Caltech, ${ }^{7} \mathrm{UMI} 3386$ CNRS / U. de Chile
}

\begin{abstract}
Edge-on, optically thick circumstellar disks have been previously imaged at subarcsecond resolution around about a dozen nearby young stellar objects. In these systems the central star is occulted from direct view, bright star image artifacts are absent, and the disk reflected light is clearly seen. Comparison of Hubble Space Telescope (HST) edge-on disk images with scattered light models has allowed key disk structural parameters and dust grain properties to be determined. Edge-on disks have been systematically undercounted to date: while $10 \%$ of young stars should statistically be occulted by their disk, the observed frequency is much less. Thus there is a significant potential for discovering and imaging new examples. Spitzer Space Telescope legacy science programs have provided the first good spectral energy distribution (SED) measurements for the previously known edge-on disks. These can be used as templates to identify new candidates in far-infrared survey datasets.

We report on the results of our HST program to image twenty-one edge-on disk candidates mostly selected from their SEDs. Eleven are well-resolved with radii ranging from 30-400 AU, nine for the first time and six showing highly collimated jets. Outstanding individual sources include a large and symmetric new template object, a highly flattened disk not accreting onto its central star, and an asymmetric disk with a misaligned jet which likely traces tidal perturbations in a binary system. Follow-up work to obtain ancillary data and perform scattered light modeling of the most symmetric disks is now being pursued. The results of this program will guide a new round of searches for these rare but important snapshots of protoplanetary disk evolution.
\end{abstract}

Keywords. Protoplanetary disks, Stars: pre-main sequence, ISM: jets and outflows

\section{Background and Previous Work}

The nearest large samples of protoplanetary disks are located in star-forming regions at distances of $\sim 150 \mathrm{pc}$. Their internal structure provides important clues to the planet formation processes, but spatial resolutions below $0.2^{\prime \prime}$ are required to resolve the relevant size scales. Scattered light imaging at $0.05^{\prime \prime}$ resolution with $H S T$ at optical wavelengths, and with ground-based adaptive optics in the near-IR, has produced the most detailed images to date of these disks (Watson et al. 2007). The reflected light of $\sim 35$ young circumstellar disks has been reported; see http://circumstellardisks.org for an updated compilation. About 15 have been detected against the bright glare of directly visible starlight (e.g., TW Hya, Krist et al. 2000; IM Lupi, Pinte et al. 2008; J1604-2130, Mayama et al. 2012). In the other cases, nearly edge-on disks have been resolved as absorption lanes bisecting an extended reflection nebulosity. In addition to these local young stars, more than thirty backlit absorption disks have been imaged at these wavelengths toward the Orion Nebula at three times greater distance (Ricci et al. 2008).

Edge-on disks offer a unique opportunity for scattered light imaging, as the bright central star is occulted from view and thus does not pose a contrast problem. Furthermore, in an edge-on system the disk's thickness can be directly seen, tracing the local 

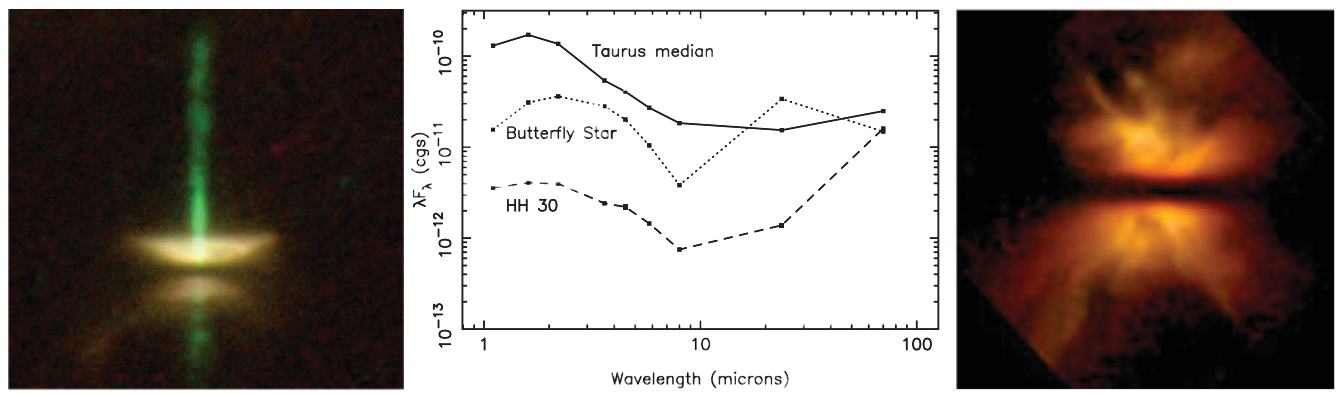

Figure 1. Left: HST/WFPC2 VRI image of the HH 30 edge-on disk (Watson \& Stapelfeldt 2004), shown in log stretch. The disk is 450 AU in diameter. Center: Spitzer SEDs of HH 30 (dashed line) and the Butterfly Star (dotted line) compared with the median Spitzer SED of young stars in the Taurus star-forming region (solid line; Rebull et al. 2010). Right: HST/NICMOS JHK image of the Butterfly Star (IRAS 04302+2247; Padgett et al. 1999), shown in log stretch. The dark lane extends $840 \mathrm{AU}$ in diameter.

disk temperature and the degree of dust settling toward the disk mid-plane. Detailed comparisons of the images with scattered light models, starting with HH 30 (Burrows et al. 1996; Wood et al. 1998; Cotera et al. 2001; Watson \& Stapelfeldt 2004; see Fig. 1), have allowed measurements of disk radii, vertical scale heights, radial flaring, and the properties of the scattering dust. Interesting variations between disks are apparent: small scale heights indicative of dust settling are seen in HK Tau B (McCabe et al. 2011), ISMlike dust grains are found in Class I sources such as the Butterfly Star (Wolf et al. 2003) and CB 26 (Sauter et al. 2009) but larger grains are required in the case of HH 30. In multiple systems disk outer radii appear to be systematically smaller (Stapelfeldt et al. 1998, 2003; Perrin et al. 2006; Neuhäuser et al. 2009). Scattered light dominates the SED of edge-on disks out to mid-infrared wavelengths (McCabe et al. 2003, Duchêne et al. 2010), indicating both high column densities and the presence of large grains. To fully reveal the diversity of young disk properties, it would be highly valuable to image additional examples.

\section{A New Target Sample}

The edge-on disks known up to now have largely been imaged by accident during companion searches or studies of Herbig-Haro jet sources. A new path to identifying these objects has been opened by the availability of sensitive far-IR surveys of nearby star-forming regions conducted by the Spitzer, Herschel, and WISE missions. The Spitzer "c2d" (Evans et al. 2009), Taurus (Rebull et al. 2010), and Gould Belt Legacy Surveys (Allen et al. 2007) have captured the SEDs of the known edge-on disks in nearby molecular clouds. In comparison to the median SED for young stars, the edge-on disk SEDs (Fig. 1) are underluminous by factors of 10-100 in the optical and near-IR (but not highly extincted), have otherwise normal far-IR and submm luminosities, and typically show distinct peaks of scattered light and thermal emission. Using these as templates, we searched through $\sim 2000$ Spitzer young star SEDs in the aforementioned surveys and identified dozens of them with similar characteristics. This list was narrowed to the 21 best-characterized and optically visible candidates. Each was observed with the Advanced Camera for Surveys (ACS) for a single orbit in our HST Cycle 19 Program 12514. Images were made in the F606W and F814W filters in order to distinguish disk reflected light (present in both bands) from jet line emission (which is nearly absent in F814W). 

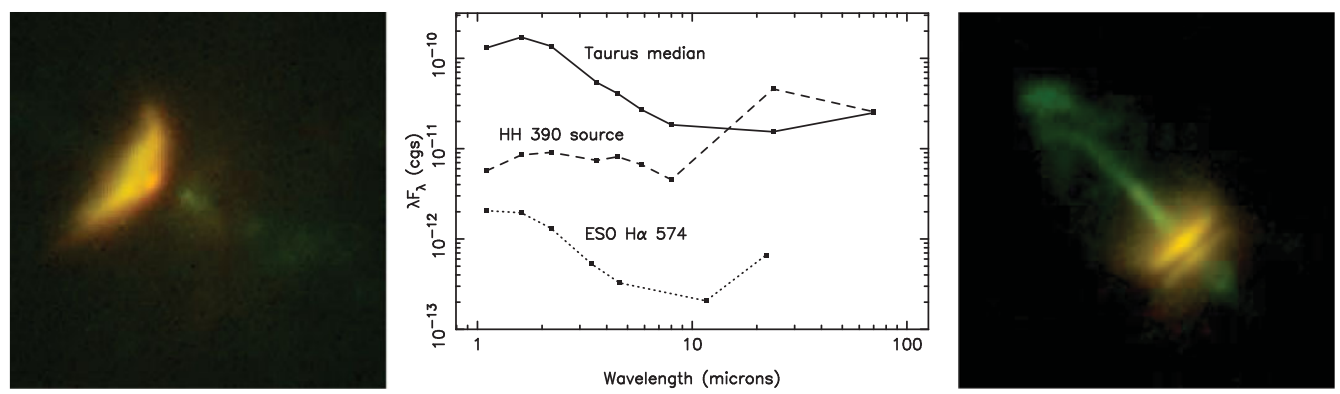

Figure 2. Left: $H S T$ /ACS RI image of the HH 390 source, shown in log stretch. The one-sided nebula is 220 AU in diameter. Center: Spitzer SED of HH 390 source (dashed line) and WISE SED of H $\alpha 574$ (dotted line) compared with the median Spitzer SED of young stars in Taurus (solid line; Rebull et al. 2010). Right: HST/ACS RI image of ESO H $\alpha 574$, shown in log stretch. The disk is $150 \mathrm{AU}$ in diameter and the outermost jet knots extend to 7.5" .

\section{HST Survey Results}

Eleven objects are resolved as edge-on disks on our survey, nine of these for the first time. Highly collimated jets are seen in six of these systems. Six other objects proved to be compact nebulae with odd or cometary morphology, and four targets appeared as point sources with no nebulosity. The latter may have suffered from confused Spitzer SEDs. Due to space limitations only a subset of the results are discussed here.

The HH 390 star is located in Taurus. Its flat SED from 1-10 $\mu \mathrm{m}$ is consistent with a scattered light disk inclined away from edge-on, and this is confirmed by the appearance of a one-sided nebula in the HST image (Fig. 2). The flared nebulosity extends perpendicular to the outflow axis with a diameter of $220 \mathrm{AU}$.

ESO H $\alpha 574$ is a very compact disk in Chamaeleon with a collimated outflow first studied by Comerón \& Reipurth (2006). The low source luminosity and almost edge-on orientation make this one of the faintest edge-on disks yet recognized. The disk is very flat and shows an unusual diffuse halo of reflected light along the base of the jet (Fig. 2).

Oph 163131.2 is a new object in Ophiuchus discovered by Evans et al. (2009). The disk is very close to edge-on with a classic double-peaked SED. It is $320 \mathrm{AU}$ in diameter and is highly flattened (Fig. 3), suggesting the possibility of vertical dust settling. There is no extended jet emission, and the star surprisingly lacks measurable $\mathrm{H} \alpha$ emission.

The HH 48 source in Chamaeleon is revealed as a $2.3^{\prime \prime}$ binary (Fig. 3). The primary component is the source of the known outflow and appears pointlike, but its faintness and the absence of strong reddening suggests it is seen in scattered light. The secondary has an edge-on disk with its own bipolar jets along $\mathrm{PA}-10^{\circ}$ and a unique internal structure. This disk is $200 \mathrm{AU}$ in diameter; the dark lane appears asymmetrically thicker on its western side; the intersection of the jet axis and the disk plane is offset $\sim 20$ AU from the center of the disk; and its jet axis is tilted $6^{\circ}$ from an axis perpendicular to the disk plane. These features, along with the outer radius $<1 / 3$ the projected binary separation, suggest tidal disturbances from the primary acting on the outer disk of the secondary.

Tau 042021.4 is a large and symmetric edge-on disk that may become a new prototype for the class. Our results for this object can be found in Duchêne et al. (these proceedings).

ESO H $\alpha 569$ is a compact flared disk with an unusually large scale height consistent with a low stellar mass. For our results on this object, see Wolff et al. (these proceedings).

The $>50 \%$ success rate of our program demonstrates that SED selection is an effective way to identify new edge-on disks for study. Our results increase the number of edge-on disks imaged with HST in nearby molecular clouds by more than $50 \%$. Several 

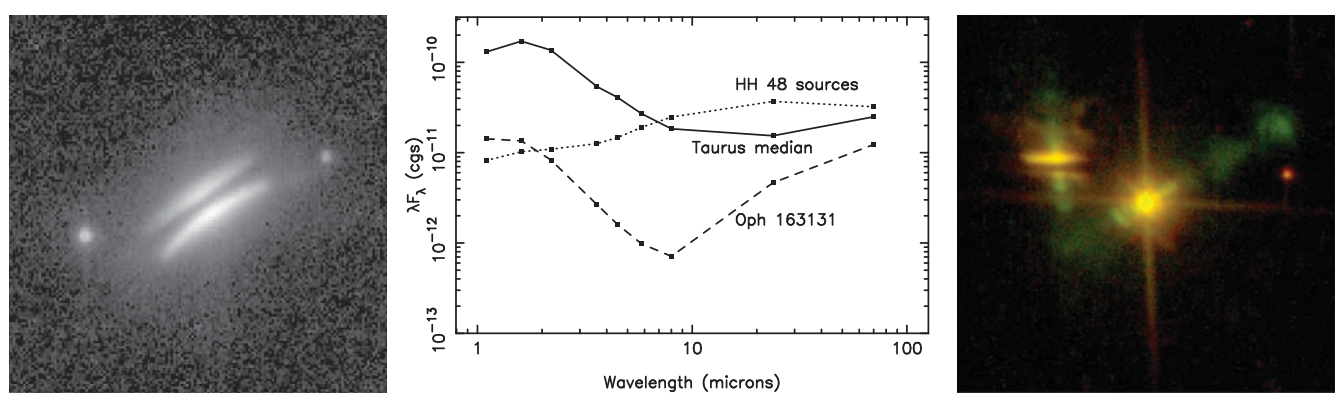

Figure 3. Left: HST/ACS R image of the edge-on disk of SSTc2dJ163131.2-242628 shown in $\log$ stretch. The disk is 320 AU in diameter. Center: Spitzer SEDs of SSTc2dJ163131.2 (dashed line) and the HH 48 system (dotted line) compared with the median Spitzer SED of young stars in Taurus (solid line; Rebull et al. 2010). Right: HST/ACS RI image of the HH 48 outflow sources, shown in log stretch. The secondary star has an edge-on disk $200 \mathrm{AU}$ in diameter.

outstanding objects were found with unique characteristics, and there are many more candidates to pursue in the Spitzer, Herschel, and WISE datasets.

Combined scattered light and SED modeling is being pursued for the most symmetric sources, with the goal of placing specific constraints on their density distributions and dust grain properties. Goals for additional datasets include expanding the wavelength range of scattered light imaging (to measure the dust lane chromaticity), filling out $\mathrm{mm} / \mathrm{submm}$ photometry, and imaging with ALMA.

\section{Acknowledgements}

This work was supported in part by HST GO grant 12514 to NASA/Goddard, UC Berkeley, and JPL/Caltech. Funding was provided by NASA, but was restricted such that the NASA and JPL co-Is were forbidden to attend this Symposium. This work was presented in oral session by G. Duchêne.

\section{References}

Allen, L., \& Spitzer Gould Belt Legacy Team 2007, BAAS, 39, 881

Burrows, C. J., Stapelfeldt, K. R., Watson, A. M., et al. 1996, ApJ, 473, 437

Comerón, F. \& Reipurth, B. 2006, A\&A, 458, L21

Cotera, A. S., Whitney, B. A., Young, E., et al. 2001, ApJ, 556, 958

Duchêne, G., McCabe, C., Pinte, C., et al. 2010, ApJ, 712, 112

Evans, N. J., et al. 2009, ApJS, 181, 321

Krist, J. E., Stapelfeldt, K. R., Ménard, F., et al. 2000, ApJ, 538, 793

Mayama, S., Hashimoto, J., Muto, T., et al. 2012, ApJ, 760, L26

McCabe, C., Duchêne, G., \& Ghez, A. M. 2003, ApJ, 588, L113

McCabe, C., Duchêne, G., Pinte, C., et al. 2011, ApJ, 727, 90

Neuhäuser, R., Krämer, S., Mugrauer, M., et al. 2009, A\&A, 496, 777

Padgett, D. L., Brandner, W., Stapelfeldt, K. R., et al. 1999, AJ, 117, 1490

Perrin, M. D., Duchêne, G., Kalas, P., \& Graham, J. R. 2006, ApJ, 645, 1272

Pinte, C., Padgett, D. L., Ménard, F., et al. 2008, A\&A, 489, 633

Rebull, L. M., Padgett, D. L., McCabe, C.-E., et al. 2010, ApJS, 186, 259

Ricci, L., Robberto, M., \& Soderblom, D. R. 2008, AJ, 136, 2136

Sauter, J., Wolf, S., Launhardt, R., et al. 2009, A\&A, 505, 1167

Stapelfeldt, K. R., Krist, J. E., Menard, F., et al. 1998, ApJ, 502, L65

Stapelfeldt, K. R., Ménard, F., Watson, A. M., et al. 2003, ApJ, 589, 410

Watson, A. M. \& Stapelfeldt, K. R. 2004, ApJ, 602, 860 
Watson, A. M., Stapelfeldt, K. R., Wood, K., \& Ménard, F. 2007, Protostars \& Planets V, 523 Wolf, S., Padgett, D. L., \& Stapelfeldt, K. R. 2003, ApJ, 588, 373

Wood, K., Kenyon, S. J., Whitney, B., \& Turnbull, M. 1998, ApJ, 497, 404

\section{Discussion}

TRIAUD: Is the misaligned jet you detected the first such occurrence?

DuCHÊNE: As far as I know, Yes, but it's also one of the best defined: the disk and the jet orientations can be estimated precisely. This is not the case for all objects. Furthermore, Right now it's not possible to know if there is a warp and if a putative inner disc would be aligned with the jet.

LIN: Are the binaries with misaligned jets physically associated?

Duchêne: Most likely. They both are members of the Cha cloud, whose population is relatively sparse, so it is not likely that they are un-related. However, no definitive evidence is available as proper motions is slow at these distances and orbital motion too slow to monitor. 